\title{
Subtle disturbances of vision after optic neuritis elicited by studying contrast sensitivity
}

\author{
R. L. Z I M M E R N, F. W C A M P B E L L, A N D \\ I. M. S . W I L K I N S O N
}

From the Department of Neurology, Addenbrooke's Hospital, and The Physiological Laboratory, Cambridge University, Cambridge

S U M M A R Y Eight patients with a subjective disorder of vision yet normal Snellen acuities after optic neuritis were shown to have an abnormal contrast sensitivity function in their affected eye. It appears that certain disorders of vision are associated with an abnormality of the contrast sensitivity function in spite of near normal visual acuity. Such an abnormality may affect pattern recognition without having an influence on Snellen acuity because of the high contrast of the latter and its predominant association with the higher spatial frequencies. Contrast sensitivity function is thus the only tool available to study those aspects of vision which have remained impervious to other subjective tests of visual function.

The determination of visual acuity in clinical practice has relied almost entirely on the use of Snellen or similar charts, in which the letters used are highly contrasted with their background and their sizes diminish from line to line so as to present a progressively smaller visual angle to the eye. Such a measurement describes only a limited aspect of visual perception, for it is common experience that the visibility of objects depends not only on their size but on their relative brightness with respect to their background. The relationship between luminosity of object and background may be expressed precisely as a contrast measurement and the ability of the visual system to see and analyse objects and patterns may be considered in terms of contrast as well as size.

The study of this relationship can be carried out conveniently using sinusoidal gratings of different contrasts and spatial frequencies (Campbell and Maffei, 1974). These are repetitive bar patterns where the luminance of the dark and light bars are varied in a sinusoidal fashion. The spatial frequency of such a pattern is defined by the number of alternating bars subtended by one degree of visual angle at the eye and the contrast by the luminance differences of dark and light bars divided by the sum of their luminances. The inter-

Address for reprint requests: Dr R. L. Zimmern, Department of Neurology, Addenbrooke's Hospital, Hills Road, Cambridge CB2 2QQ.

Accepted 26 October 1978 action of size and contrast in the human visual system may be studied psychophysically by using such gratings to determine the threshold contrast necessary for grating detection at different spatial frequencies. The reciprocal of the threshold contrast is known as the contrast sensitivity and the plot of contrast sensitivity against spatial frequency is the contrast sensitivity function. Figure 1 shows a grating varying in spatial frequency along the horizontal axis and varying in contrast along the vertical axis. The junction between the visible and invisible parts of the grafting defines the contrast sensitivity function.

The importance of contrast sensitivity is related to modern ideas concerning mechanisms of pattern recognition. Fourier showed that any waveform could be separated into sinusoidal components of different frequencies and amplitudes. His theorem was applied successfully by Helmholtz to the physiology of hearing and allowed an understanding of how complex sounds might be analysed into their respective simple frequency components. Only in recent years has such an analysis been applied to the visual system (Campbell, 1973).

Microelectrode studies have shown that cells exist in the visual system which respond to sinusoidal gratings of a limited range of spatial frequencies (Enroth-Cugell and Robson, 1966; Cleland et al., 1971; Campbell et al., 1969). These studies, which are now extensive, have enabled a theory of perception based on the concept of fre- 


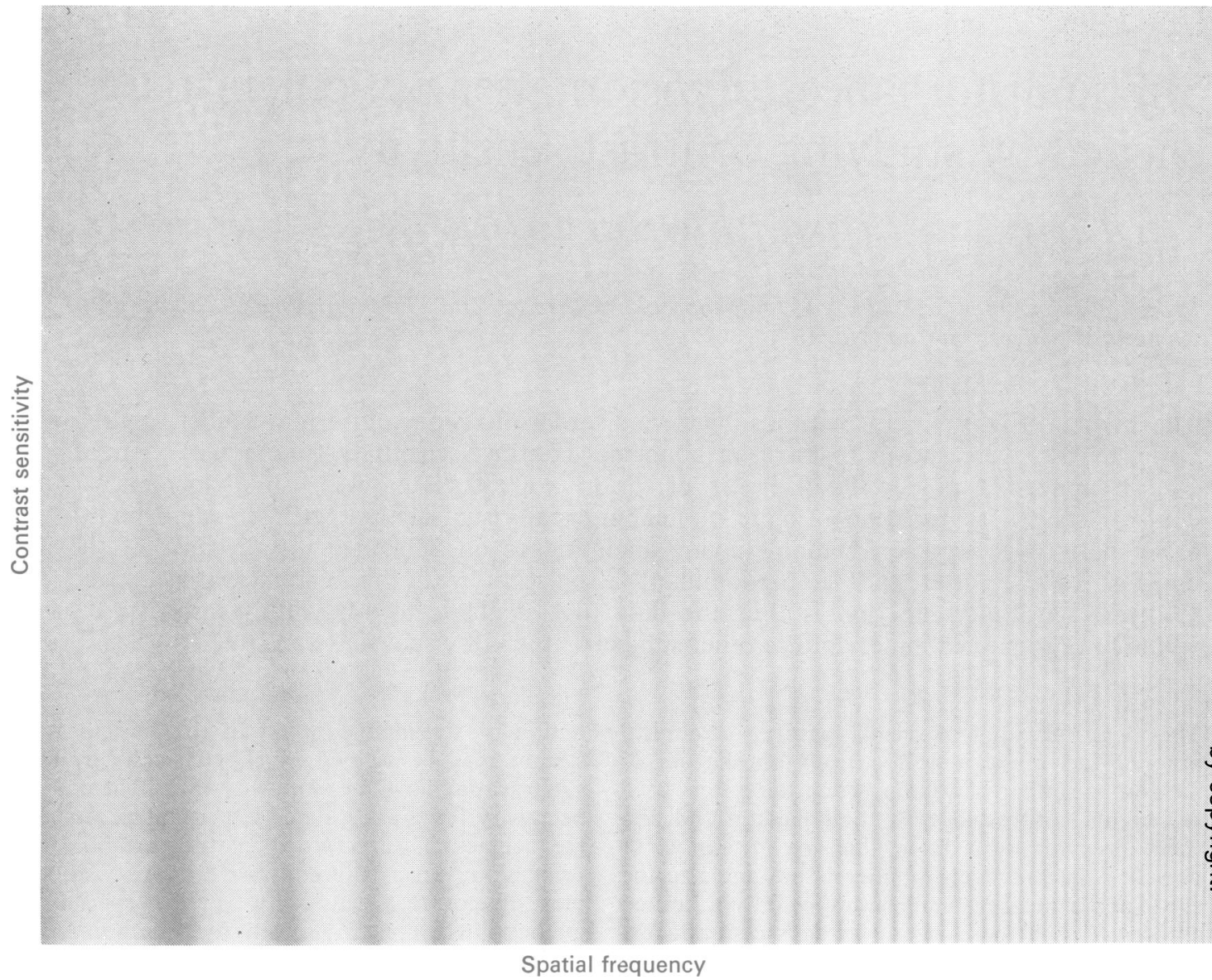

Fig. 1 Grating with spatial frequency varying along horizontal axis and contrast sensitivity along vertical axis. The junction between the visible and invisible parts of the grating defines the contrast sensitivity function.

quency specific channels in the visual pathways. The application of Fourier theory was to suggest that complex visual information could be carried in these frequency specific channels in much the same way that complex harmonies in the domain of hearing are carried in the auditory system by similar frequency channels. In man, the existence of these channels has likewise been shown by the use of appropriate psychophysical techniques. Experiments in which contrast sensitivity functions were constructed using square-wave, rectangular, and saw-tooth rather than sinusoidal gratings have produced results to be as calculated by Fourier theory (Campbell and Robson, 1968).

The normal contrast sensitivity function may, therefore, be seen as the envelope of the turning curves of the different spatial frequency specific channels with respect to their ability to respond to different contrasts. Those channels with a frequency of approximately $3 \mathrm{c} / \mathrm{deg}$ are seen to be most sensitive while channels on either side of this frequency are seen to be progressively less sensitive. Damage to the different spatial frequency channels will lower their sensitivities and will be detected as an abnormality of the contrast sensitivity function. Abnormal vision may be construed to result from damage to some or all the spatial frequency channels if the concepts of Fourier theory are followed. Such abnormal vision may be detected as an abnormal contrast sensitivity function but need not result in an abnormal Snellen acuity.

The relationship between the Snellen acuity and the contrast sensitivity function is complex but 
appears to be concerned with mechanisms at the higher end of the spatial frequency range. Thus abnormalities of the Snellen acuity would not be seen if low or medium frequency channels were damaged or if the rise in the contrast theshold (corresponding to a reduction in contrast sensitivity) in the high frequency channels did not exceed the high contrast of the Snellen figures.

An abnormality in the contrast sensitivity function has been shown in a heterogeneous group of patients with cerebral lesions and a complaint of blurred vision, (Bodis-Wollner and Diamond, 1976) and also in a number of patients with multiple sclerosis (Regan et al., 1977) some of whom showed no abnormality of visual function on standard clinical tests. This preliminary communication reports a study of eight patients with optic neuritis or multiple sclerosis who complained of a disturbance of vision in the presence of good Snellen acuities.

\section{Patients and methods}

Eight patients took part in the study. Six had welldocumented histories of optic neuritis in one eye and one had at different occasions such episodes involving each of her eyes in turn. All seven had recovered from the acute episode in that their visual acuity was $6 / 9$ or better but all still complained of some subjective disturbance of vision in the affected compared with the unaffected eye. Of these seven patients six had other symptoms and signs of multiple sclerosis. The eighth patient presented with a paraparesis but had complained of a visual disturbance in his left eye over the preceding three years in spite of normal Snellen acuities. Control subjects were members of the Neurology and EEG Departments at Addenbrooke's Hospital.

In all these patients visual acuity was assessed by the use of Snellen charts. Colour vision was determined using Ishihara pseudo-isochromatic plates, and four mistakes or greater were taken to be consistent with an abnormality of colour perception. The appearance of the optic discs was noted and a relative afferent pupillary defect was detected by the use of the "swinging torchlight test" (Levitin, 1959). Visual evoked potentials were obtained using the method of Halliday et al. (1972). The results of these ophthalmic investigations are shown in the Table.

Contrast sensitivity was determined using a method based on that of Schade (1956). Vertically orientated stationary sinusoidal gratings were displayed on the screen of a cathode-ray tube using a waveform generator. With suitable synchronisation of the time base different spatial frequencies could be displayed. The contrast of the grating pattern was varied by adjusting the modulation voltage using a logarithmic step attenuator. Contrast was defined as $L_{\max }-L_{\min } / L_{\max }+L_{\min }$ where $L_{\max }$ and $L_{\min }$ are the luminances of the light and dark bars respectively. The screen was set up with a base contrast of 0.33 with zero attenuation. The mean screen luminance was $100 \mathrm{Cd} / \mathrm{m}^{2}$ and was independent of the contrast and of the spatial frequency of the grating being displayed. The screen of the cathode-ray tube was covered by a piece of white cardboard measuring $750 \times 500 \mathrm{~mm}$ and having a central aperture $250 \times 200 \mathrm{~mm}$. No special lighting was directed at the cardboard and experiments were conducted in conditions of ordinary room lighting which was kept constant from experiment to experiment. At spatial frequencies of $2 \mathrm{c} / \mathrm{deg}$ or above, measurements were taken at a distance of $2280 \mathrm{~mm}$, and at $1 \mathrm{c} / \mathrm{deg}$ or below at $570 \mathrm{~mm}$. The spatial frequencies used were $8 \mathrm{c} / \mathrm{deg}, 6 \mathrm{c} / \mathrm{deg}, 20 \mathrm{c} / \mathrm{deg}, 4 \mathrm{c} / \mathrm{deg}, 10 \mathrm{c} / \mathrm{deg}$, $2 \mathrm{c} / \mathrm{deg}, 15 \mathrm{c} / \mathrm{deg}, 1 \mathrm{c} / \mathrm{deg}, 0.4 \mathrm{c} / \mathrm{deg}, 0.2 \mathrm{c} / \mathrm{deg}$, and $0.6 \mathrm{c} / \mathrm{deg}$, all presented in the order shown.

No formal refraction was carried out but trial lenses ranging from -0.5 to +0.5 dioptres were used if the patient thought subjectively that these improved acuity. Contrast thresholds were determined by the subject who adjusted the attenuator until a contrast was reached at which some form

Table Results of ophthalmic investigations

\begin{tabular}{|c|c|c|c|c|c|c|}
\hline Patient & $\begin{array}{l}\text { Age } \\
(y r)\end{array}$ & $\begin{array}{l}\text { Visual acuity } \\
\text { (affected eye) }\end{array}$ & Colour vision & $\begin{array}{l}\text { Afferent pupillary } \\
\text { defect }\end{array}$ & Pallor & $\begin{array}{l}\text { Visual evoked } \\
\text { response }\end{array}$ \\
\hline $\begin{array}{l}\text { HR } \\
\text { CH } \\
\text { CB } \\
\text { MJ } \\
\text { SS } \\
\text { BG } \\
\text { VA } \\
\text { MD }\end{array}$ & $\begin{array}{l}50 \\
38 \\
21 \\
30 \\
31 \\
45 \\
29 \\
23\end{array}$ & $\begin{array}{l}6 / 9 \\
6 / 6 \\
6 / 6 \\
6 / 6 \\
6 / 6 \\
6 / 6 \\
6 / 6 \\
6 / 6\end{array}$ & $\begin{array}{l}\text { Abnormal } \\
\text { Normal } \\
\text { Normal } \\
\text { Abnormal } \\
\text { Abnormal } \\
\text { Abnormal } \\
\text { Normal } \\
\text { Normal }\end{array}$ & $\begin{array}{l}+ \\
\text { Equivocal } \\
+ \\
+ \\
\text { Equivocal } \\
+ \\
\text { No } \\
\text { No }\end{array}$ & $\begin{array}{l}+ \\
\text { No } \\
+ \\
+ \\
\text { Equivocal } \\
\text { Equivocal } \\
+ \\
\text { No }\end{array}$ & $\begin{array}{l}\text { Delayed } \\
\text { Delayed } \\
\text { Delayed } \\
\text { Delayed } \\
\text { Delayed } \\
\text { Delayed } \\
\text { Delayed } \\
\text { Delayed }\end{array}$ \\
\hline
\end{tabular}

*The visual acuity of the "unaffected" eye was $6 / 6$ in all the patients. 
of stimulus could just be detected on the screen. Ten observations were made at each spatial frequency, and the results were fed into a PDP8 computer which derived, from the attenuation voltage and the base contrast, the mean contrast sensitivity.

\section{Results}

The contrast sensitivity functions of five control subjects were obtained from which a mean control curve with $99 \%$ confidence limits was derived. The contrast sensitivity function of each of the patients' eyes was individually compared with the control function. This was done by plotting, at each spatial frequency, the ratio of the test contrast sensitivity to that of its control. This resultant attenuation function has been labelled, by Bodis-Wollner, a visuogram.

Figure 2 shows the normal contrast sensitivity function derived from the five control subjects with
$1 \%, 5 \%, 95 \%$ and $99 \%$ confidence limits. The dotted contrast sensitivity function is that of the affected eye of one patient (MJ) and serves as an example of an abnormal contrast sensitivity function. Above this is the visuogram for this patient plotted by taking the ratio of her contrast sensitivity function to that of the mean control curve. Figure 3 shows the visuograms of the eight patients. The dotted line is the visuogram from the affected eye and the continuous line is the visuogram from the "unaffected" eye. The line bounding the shaded area represents the first percentile of the normal contrast sensitivity function expressed in the form of the visuogram.

It can be seen that the visuograms of the unaffected eyes of all patients except one (SS) lay within or above the shaded area. She had multiple sclerosis and was the most severely affected of the patients tested. She also made three errors in the Ishihara plates with her unaffected eye while no

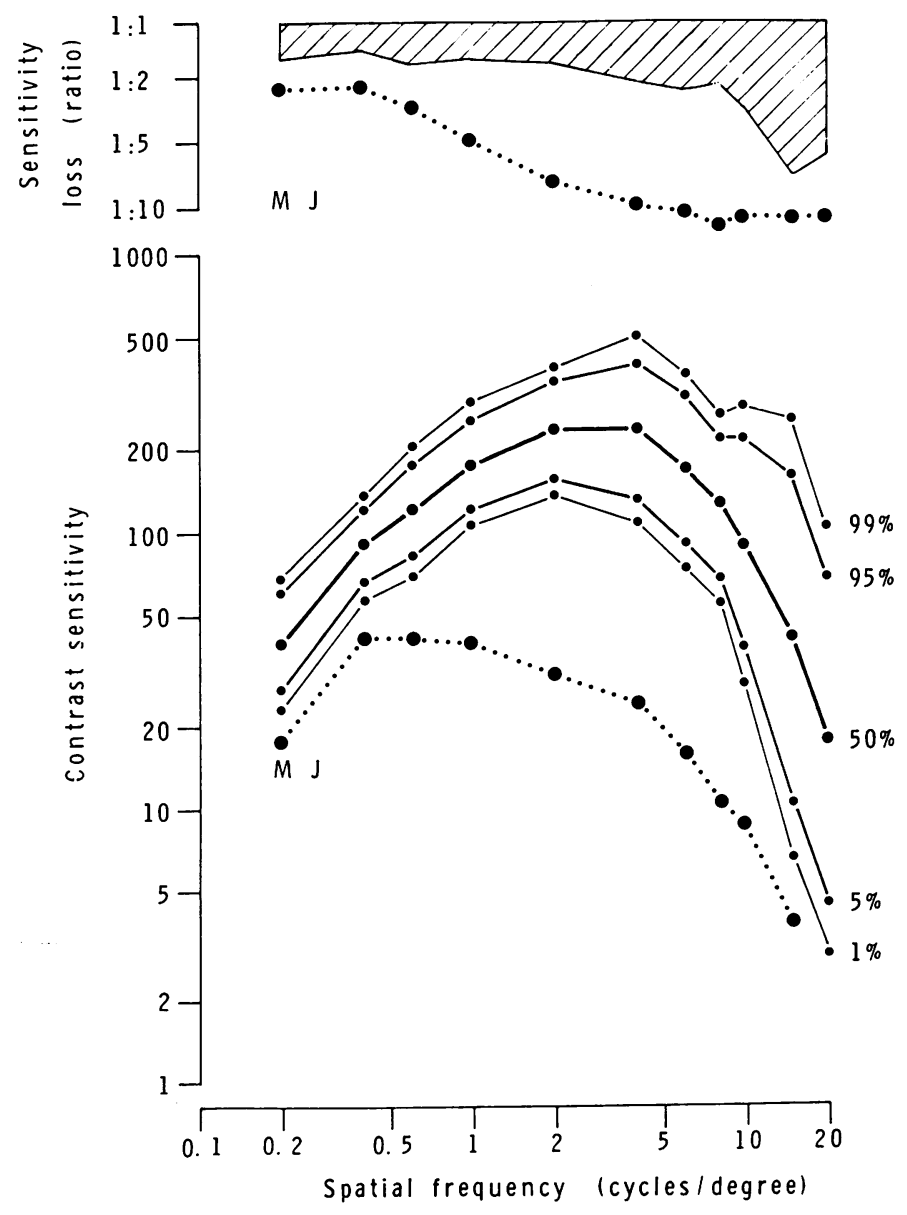

Fig. 2 Mean contrast sensitivity function from five normal subjects plotted as the 50 th percentile with $95 \%$ and $99 \%$ confidence limits plotted as first, fifth, 95th and 99th percentiles. Dotted curve is the contrast sensitivity function for the affected eye of subject $M J$. The visuogram above shows the ratio of MJ's curve to that of the mean control contrast sensitivity function. 


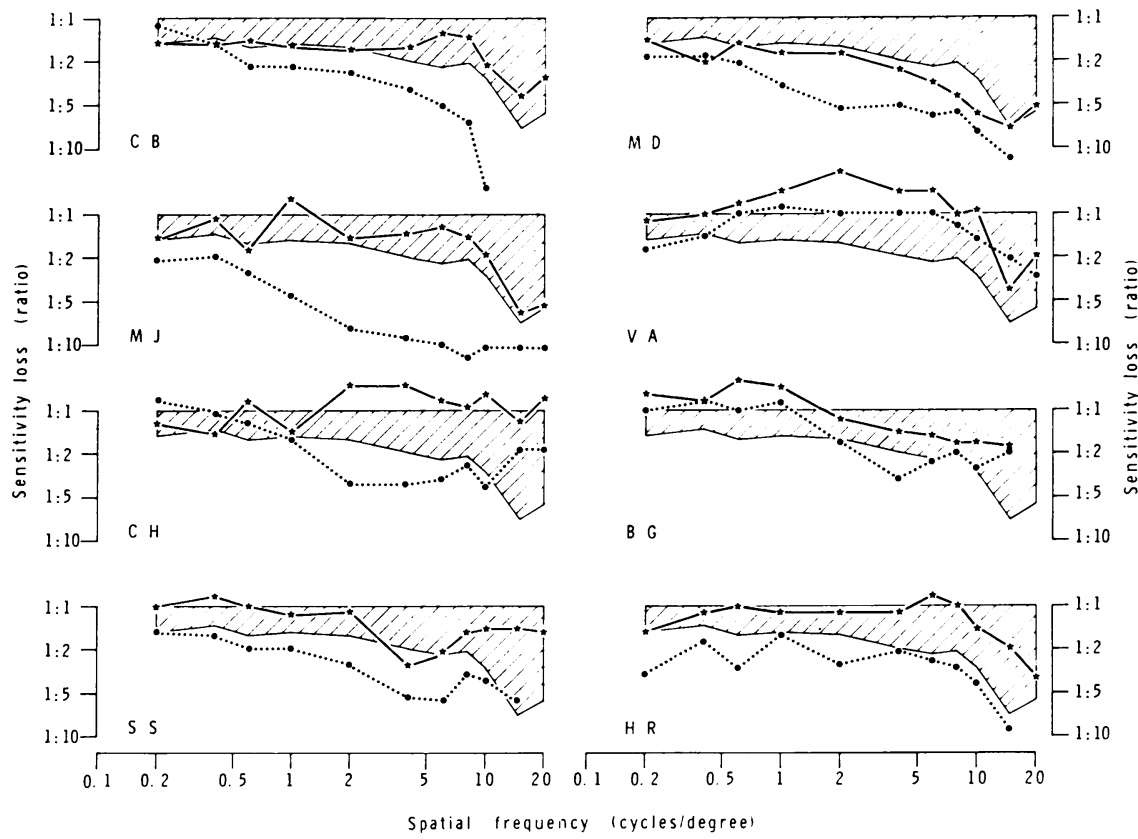

Fig. 3 Visuograms of eight patients with optic neuritis. $\bigcirc=$ right eye $\star=$ left eye. Dotted line represents the eye involved by optic neuritis. Continuous line represents the "unaffected" eye. Shaded area represents the first percentile of the normal contrast sensitivity function plotted as a visuogram.

mistakes were made by the unaffected eyes of the other patients. One can, therefore, conjecture that she had subclinical involvement of her "unaffected" optic nerve. The visuograms of both the affected and unaffected eyes of patient VA lay within the shaded area but it is clear that the visuogram from the affected eye was constantly below that from the unaffected eye. Patient MD, who had optic neuritis in both eyes, had bilaterally abnormal visuograms.

\section{Discussion}

Our results confirm those of Regan and his colleagues (1977) that there are patients with multiple sclerosis who have normal visual acuities and yet who complain of imperfect or "washed out" vision and that these patients may have an abnormal contrast sensitivity function. Their patients fell into five groups: (1) those with no abnormality in their contrast sensitivity function; (2) those with a similar loss at all spatial frequencies; (3) those with a preferential loss at high or at medium and high spatial frequencies; (4) those with a loss restricted to medium spatial frequencies; (5) those with a restricted low spatial frequency loss. Our limited study shows examples of all these groups except the fifth. Regan et al. (1977) do not give any details of the neuroophthalmological findings in their patients but entitle their paper "Visual acuity and contrast sensitivity in multiple sclerosis-hidden visual loss. An auxiliary diagnostic test." Our data, summarised in the Table, show that all our patients had some form of neuro-ophthalmological abnormality to point to an optic nerve disorder. More information is necessary to establish whether studies of the contrast sensitivity function do constitute an auxiliary diagnostic test in showing up an abnormality of optic nerve function not shown by the other clinical tests.

It does appear from the results of this study and others that certain disorders of vision are associated with an abnormality of the contrast sensitivity function in spite of near normal visual acuities. If the visual cortex acts as a spatial frequency analyser, as has been suggested (Maffei and Fiorentini, 1972, 1973), an abnormal contrast sensitivity function reflecting disturbances in certain spatial frequency channels will lead to faulty visual information processing. Such an abnormality may greatly affect pattern recognition yet not have an influence on Snellen acuity because of the high contrasts of the latter and its predomi- 
nant association with the higher spatial frequencies. The contrast sensitivity function is thus the only tool available to study those aspects of vision, in particular low and medium frequency channels and patterns of low contrast, which have remained impervious to hitherto available tests of visual function.

We believe that contrast sensitivity studies hold great promise in this respect, not only as a possible diagnostic aid but also for the relevance that abnormal findings may have to an understanding of normal visual physiology.

We wish to thank Dr M. F. T. Yealland for permission to study patients under his care, Dr Robert Hess and Dr A. P. Ginsburg for helpful discussion, Miss Peggy Hall and the staff of the EEG Department, Addenbrooke's Hospital, and Mr Clive Hood of the Physiological Laboratory, Cambridge for technical assistance.

\section{References}

Bodis-Wollner, I., and Diamond, S. P. (1976). The measurement of spatial contrast sensitivity in cases of blurred vision associated with cerebral lesions. Brain, 99, 695-710.

Campbell, F. W. (1973). The transmission of spatial information through the visual system. In Neurosciences Third Study Programme, p. 95. Edited by F. Worden and F. O. Schmitt. MIT Press: Cam- bridge, Massachusetts.

Campbell, F. W., Cooper, G. F., and Enroth-Cugell, C. (1969). The spatial selectivity of the visual cells of the cat. Journal of Physiology, 203, 223-235.

Campbell, F. W., and Maffei, L. (1974). Contrast and spatial frequency. Scientific American, 231, 106-114.

Campbell, F. W., and Robson, J. G. (1968). Application of Fourier analysis to the visibility of gratings. Journal of Physiology, 197, 551-566.

Cleland, B. G., Dubin, M. W., and Levick, W. R. (1971). Sustained and transient neurones in the cat's retina and lateral geniculate nucleus. Journal of Physiology, 217, 473-496.

Enroth-Cugell, C., and Robson, J. G. (1966). The contrast sensitivity of retinal ganglion cells of the cat. Journal of Physiology, 187, 517-552.

Halliday, A. M., McDonald, W. I., and Mushin, J. (1972). Delayed visual evoked response in optic neuritis. Lancet, 1, 982-985.

Levitin, P. (1959). Pupillary escape in disease of the retina or optic nerve. Archives of Ophthalmology, 62, 768.

Maffei, L., and Fiorentini, A. (1972). Processes of synthesis in visual perception. Nature, 240, 479-481.

Maffei, L., and Fiorentini, A. (1973). The visual cortex as a spatial frequency analyser. Vision $R e$ search, 13, 1255-1267.

Regan, D., Silver, R., and Murray, T. J. (1977). Visual acuity and contrast sensitivity in multiple sclerosis: hidden visual loss. Brain, 100, 563-579.

Schade, O. H. (1956). Optical and photoelectricalo analog of the eye. Journal of the Optical Societyo of America, 46, 721-739. 